\title{
YAP-1 Promotes Tregs Differentiation in Hepatocellular Carcinoma by Enhancing TGFBR2 Transcription
}

\author{
Ye Fan ${ }^{a, b}$ Yangjuan Gao ${ }^{a, b}$ Jianhua Rao ${ }^{a, b}$ Feng Zhang ${ }^{a, b}$ Ke Wanga,b \\ Chuanyong Zhang ${ }^{a, b}$
}

${ }^{a}$ Key Laboratory on Living Donor Liver Transplantation, Ministry of Health, ${ }^{b}$ Department of Liver Surgery, 'Department of Radiology. First Affiliated Hospital of Nanjing Medical University, Nanjing, Jiangsu, China

\section{Key Words}

YAP-1 - Treg • TGFBR2 • TEADs • HCC

\begin{abstract}
Background/Aims: Immunosuppression is one of the hallmarks of cancer; however, its molecular mechanism remains unknown. In the present study, we sought to investigate the expression and activation of yes-associated protein 1 (YAP-1) and its roles in T cells within hepatocellular carcinoma (HCC). Methods: The expression and activation of YAP-1 were accessed by real-time PCR, immunohistochemistry staining, western blot, and flow cytometry. The potential regulation effect of YAP-1 on Regulatory T cells (Tregs) differentiation was predicted using bioinformatics tools and verified by in vitro studies. Results: Significant overexpression and activation of YAP-1 was detected within peripheral blood mononuclear cells and showed positive linear correlation to Treg percentage; it may serve as a valuable indicator of a bad prognosis. Using in vitro studies, we found that overexpression and activation of YAP-1 can promote naïve T cell polarization stimulation to Tregs by increasing the expression of TGFBR2. The YAP-1/TEADs DNA binding site was spotted within the promoter region of TGFBR2 and related to its transcription activity. YAP-1 acted as a co-activator of TGFBR2 transcription by binding directly to the TGFBR2 promoter through TEADs. Conclusion: Overexpression and activation of YAP-1 in HCC T cells can induce immunosuppression by promoting Treg differentiation via transcriptional enhancement of TGFBR2.
\end{abstract}

\section{Introduction}

(C) 2017 The Author(s) Published by S. Karger AG, Basel

The expression tumor microenvironment (TME) was recently termed to describe the environment surrounding tumor cells [1-3]. The TME is a cellular microenvironment where the tumor cells live within contributing the tumor cells for self-repairing and escaping from

F. Ye and G. Yangjuan contribute equally to this research.

Wang Ke MD PhD

and Zhang Chuanyong MD PhD
300 Guangzhou Rd, Nanjing, Jiangsu, 210029 (China)

E-Mail drwangk110@gmail.com/zhangcyong888@126.com

\section{KARGER}


immune surveillance [4]. It consists of a variety of cells and factors associated with stromal cells of hematopoietic and nonhematopoietic lineages such as: T-cells, B-cells, natural killer cells, and myeloid cells $[5,6]$. Within all categories of component cells, the immune infiltrated lymphocytes are not only a good extrapolative factor in immune surveillance, but also help to improve the efficiency of conventional chemotherapy [7, 8]. Emerging evidence indicates that T regulatory cells (Tregs) and myeloid-derived suppressor cells can be responsible for inhibiting host T-cell activity against tumor-associated antigens and that they inhibit the effectiveness of anti-cancer immunotherapeutic approaches [9].

Tregs express the markers CD4, Foxp3, and CD25, and are thought to be derived from the same lineage as naïve CD4 cells [9]. TGF- $\beta$ is essential for Tregs to differentiate from naïve CD4+ cells and is important in maintaining Treg homeostasis. The Treg percentage was regarded as a critical factor in determining the prognosis of cancer, and its related immunosuppressive effect was also considered as one of the disadvantages of immunotherapy [10]. Reports concerning the roles of Tregs in liver cancer started to appear about 10 years ago, Yang et al. indicated that hepatocellulat carcinoma (HCC) Tregs distributed mainly in the marginal region of the tumor might play a critical role in controlling CD8+ cytotoxic T-cell activity and thus contribute to the progression of HCC [11]; also the circulating and tumor-infiltrating Tregs were associated with high mortality and reduced survival time of HCC patients [12-14]. However, the reasons for increasing Treg levels within HCC have not been completely identified.

Yes-associated protein (YAP) is a protein that acts as a transcriptional regulator by activating the transcription of genes involved in cell proliferation and of apoptosissuppressing genes [15]. Within the tumor, YAP-1 itself lacks DNA-binding capacity, and can act as a co-activator when combined with the TEAD family as a transcriptional coactivator [15-17]. YAP-1 and its close paralog, TAZ (WWTR1), are the main effectors of the Hippo tumor suppressor pathway [15]. When the pathway is activated, YAP-1 and TAZ are phosphorylated on a serine residue and sequestered in the cytoplasm by 14-3-3 proteins [18]. Almost all of the roles of YAP-1 have been identified from tumor cells; a little knowledge has been identified concerning its roles in the immune cell. However, several studies have revealed its role in immunosuppression $[19,20]$, it is meaningful to investigate its role in Treg development in HCC.

Therefore, in our present study, we investigated the activation of Hippo signaling in tumor-infiltrating T cells, especially the role of YAP-1, where particular attention was paid to its regulatory effect on Treg differentiation, proliferation, and functions.

\section{Materials and Methods}

\section{Patients}

A total of 152 HCC cases and sex plus age-matched 91 controls obtained from Jiangsu Province Hospital, were included in this study. Patients were consecutively recruited between February 2008 and January 2015. The detailed information of HCC patients and the healthy controls was listed in Table 1. All cases are incident ones during enrollment of the current case-control study. The diagnosis of all patients was histological confirmed. A face-to-face questionnaire was administered to collect demographic data. All participants have provided their written informed consents to participate in this study. This study was approved by the Institutional Review Board of Jiangsu Province Hospital.

Real-time PCR assay

Real-time polymerase chain reaction (RT-PCR) was performed to determine whether the mutation changed the expression level of Foxp3 and Tgfbr2. The primers used for amplification were forward primer: GTGGCCCGGATGTGAGAAG, and reverse primer: GGAGCCCTTGTCGGATGATG for Foxp3, forward primer: ACGGCGTTACAGTGTTTCTG, and reverse primer: GCACATACAAACGGCCTATCTC for Tgfbr2 and forward primer: TGTGGGCATCAATGGATTTGG, reverse primer: ACACCATGTATTCCGGGTCAAT for GAPDH. The amplification conditions were $95^{\circ} \mathrm{C}$ for 10 minutes, followed by 40 cycles of $95^{\circ} \mathrm{C}$ for 30 seconds, $55^{\circ} \mathrm{C}$ for 


\section{Cellular Physiology Cell Physiol Biochem 2017;41:1189-1198 \begin{tabular}{l|l|l} 
and Biochemistry Published onIIne: IVIarch 06, 2017 & $\begin{array}{l}\text { (c) } 2017 \text { The Author(s). Published by S. Karger AG, Basel } \\
\text { www.karger.com/cpb }\end{array}$
\end{tabular}}

40seconds, and $72^{\circ} \mathrm{C}$ for 30 seconds, and finally $4^{\circ} \mathrm{C}$ for 30 minutes for cooling by the 384-well ABI 7900HT Real-Time PCR System. The data was processed by using $2{ }^{-\Delta \Delta c t}$ methods, and to normalize the results, all relative expression of YAP-1 was multiplied by 100 , and TGFBR2 was multiplied by 10 .

Transduce of $T$ cells and polarization stimulation of Tregs

The $\mathrm{T}$ cells isolated from the healthy controls were isolated by using magnetic bead (Thermofisher, CA) and transduced by using lentiviral-based gene delivery. The shRNA sequence targeting YAP-1 and TGFBR2 were AGGTCCTCTTCCTGATGGATGGGAA for YAP1 and CAGCTGTAATAGGACTGCCCATCCA for TGFBR2. For polarization stimulation, T cells
Table 1. Clinical characteristic of HCC patients and cancer-free controls. *Two-sided chi-square test for either genotype distributions or allele frequencies between cases and controls

\begin{tabular}{|c|c|c|c|c|c|}
\hline \multirow{2}{*}{ Variables } & \multicolumn{2}{|c|}{ Cases $(n=152)$} & \multicolumn{2}{|c|}{ Controls $(n=91)$} & \multirow{2}{*}{$P^{*}$} \\
\hline & $N$ & $\%$ & $N$ & $\%$ & \\
\hline \multicolumn{6}{|c|}{ Age (years) } \\
\hline$\leq 50$ & 71 & $46.71 \%$ & 49 & $53.85 \%$ & 0.597 \\
\hline$>50$ & 81 & $45.11 \%$ & 42 & $46.15 \%$ & \\
\hline \multicolumn{6}{|l|}{ Gender } \\
\hline Male & 76 & $50.00 \%$ & 45 & $49.45 \%$ & 1.000 \\
\hline Female & 76 & $50.00 \%$ & 46 & $50.55 \%$ & \\
\hline \multicolumn{6}{|c|}{ Tumor Size $(\mathrm{cm})$} \\
\hline$\leq 5 \mathrm{~cm}$ & 73 & $48.03 \%$ & & & \\
\hline$>5 \mathrm{~cm}$ & 79 & $51.97 \%$ & & & \\
\hline \multicolumn{6}{|c|}{ Differentiation } \\
\hline Well & 47 & $30.92 \%$ & & & \\
\hline Moderate & 54 & $35.53 \%$ & & & \\
\hline Poor & 51 & $33.55 \%$ & & & \\
\hline \multicolumn{6}{|l|}{ Metastasis } \\
\hline Yes & 69 & $45.39 \%$ & & & \\
\hline No & 83 & $54.61 \%$ & & & \\
\hline
\end{tabular}

were treated with TGF- $\beta(2 \mathrm{ng} / \mathrm{mL})$ and maintained in T cell culture medium for 7 days.

\section{Immunohistochemistry}

Sections were de-paraffinized and followed by rehydration steps through a graded ethanol series and distilled water, and then were treated with $3 \% \mathrm{H}_{2} \mathrm{O}_{2}$ in methanol for 30 min to block the endogenous peroxidase activity. The sections were rinsed in phosphate-buffered saline (PBS) twice, 5 min each time and incubated with 10\% normal goat serum for $30 \mathrm{~min}$ to block non-specific antibody binding. After washing, the samples were incubated with primary anti-rabbit antibody Foxp3 (ab16901) TGFBR2 (ab78419) and YAP-1 S127 (ab76252) purchased from Abcam at $4^{\circ} \mathrm{C}$ overnight, and then washed with PBS for three times and then incubated with secondary antibodies. After that, the sections were stained with DAB according to manufacturer's protocols and mounted and photographed using a digitalized microscope camera (Nikon, Tokyo, Japan).

\section{Dual-luciferase reporter assay}

For luciferase activity analysis, Jurkat cells were cotransfected with 100ng of luciferase reporter constructs (pGL4 Luciferase Reporter Vector, Promega, WI) 5ng of the Renilla control plasmid and 10 pmol of wild-type and a mutant plasmid with $1 \mu$ lipofectamine 2000 according to the manufacturer's instructions (Invitrogen, NY, USA). After incubation for $48 \mathrm{~h}$, we carried out the luciferase assay using the luciferase reporter assay system (Promega, Madison, WI) according to the manufacturer's protocol. Measurements of luminescence and absorbance of $\beta$-gal were performed on a luminometer (Glomax 20/20; Promega). Three independent experiments were performed in triplicate.

Western blot and Co-Immunoprecipitation (Co-IP) Assay

For western blotting, proteins were extracted from tissues or cultured cells using RIPA buffer containing phenylmethanesulfonylfluoride(PMSF) (Beyotime, Nantong, China). An equal amount of proteins (100ug) were separated by 7.5\%/12.5\% sodium dodecyl sulfate polyacrylamide gel electrophoresis (SDS-PAGE) and transferred to polyvinylidene fluoride (PVDF) membrane. Primary polyclonal antibodies targeting YAP-1 (ab71153), YAP-1 s127 (ab76252) and Foxp3 (ab16901) were purchased from Abcam (Cambridge, MA). The secondary antibodies were anti-rabbit or anti-mouse HRP-linked were purchased from Santa Cruz Biotechnology (CA, USA). The blots were developed using ECL reagent (Millipore, MASS, USA). An equal amount of protein loading in each lane was confirmed using $\beta$-actin antibody. Image software quantified the integrated density of the band.

For Co-IP assay, protein was extracted from Jurkat by using IP Lysis/Wash Buffer. The YAP/TEADs complex is then captured and precipitated by both YAP-1 (ab71153) and TEADs (ab197589) Antibody by using a Dynabeads $\circledR$ Protein A Immunoprecipitation Kit (Thermofisher, CA), and further processed for YAP and TEADs detection. 


\section{Cellular Physiology Cell Physiol Biochem 2017;41:1189-1198 \begin{tabular}{l|l|l} 
and Biochemistry Published onIIne: IVIarch 06, 2017 & $\begin{array}{l}\text { (c) } 2017 \text { The Author(s). Published by S. Karger AG, Basel } \\
\text { www.karger.com/cpb }\end{array}$
\end{tabular}}

Chromatin immunoprecipitation (ChIP) assay

ChIP was performed using a commercially available EZ-ChIP Chromatin Immunoprecipitation (ChIP) Assay Kit (Millipore) according to manufacturer's instructions. DNA-bound protein was immunoprecipitated using both anti-YAP (ab71153, Abcam) and IgG (Abcam) as a negative control. For quantification of coprecipitated DNA, samples were then subjected to amplification by employing primers (Forward primer: GAGGCTATTTGGGGGTGTGT and reverse primer: TCAACTTCAGCATTCCCCCG) which amplified the promoter region (186 bp) of the $T g f b r 2$ promoter.

In vitro stimulation of Tregs and Flow cytometry (FCM)

For in vitro generation of Tregs, naïve T cells was isolated by human naïve T cell isolation kit II (Miltenyi, Koln, Germany), and further stimulated with human recombined human TGF- $\beta$ (1ng/mL) (Peprotech, NJ ) for 7 days. Dead cells were excluded based on staining with Live/Dead fixable dye. After that, cells were, fixed, permeabilized with IntraPrep reagent (BD Pharmingen, CA, USA), and then stained with fluorochromeconjugated labeled antibodies including CD4 (561833), CD25 (557192), Foxp3 (560082) (BD Pharmingen, CA, USA). The Tregs were determined gated with CD4 and further defined as CD25 and Foxp3 positive cells. Data were acquired on BD FACSVerse ${ }^{\mathrm{TM}}$ flow cytometer (BD Pharmingen, CA, USA).

Statistical analysis

Differences between cases and controls were evaluated by the Student's t-test for continuous variables and the $\chi 2$ test for categorical variables. The two values correlation was assessed by Linear correlation analysis and tested $\mathrm{F}$ test. The overall survival in different groups was analyzed by using Kaplan-Meier curve. All statistical analyses were performed using Statistical Package for Social Sciences software.

\section{Results}

YAP-1 is overexpressed in the peripheral T cells and acts as an index for a bad prognosis of HCC

In order to investigate the expression of YAP-1 in the T cells of human HCC, the peripheral blood mononuclear cells (PBMCs) were isolated from 152 HCC patients and 91 healthy controls, and the expression of YAP-1 was measured by real-time PCR. We found that YAP-1 was significantly overexpressed in HCC PBMCs (Fig. 1A). There are two major component cells within PBMCs, T cells, and monocytes. YAP-1 expression was measured in both cell types isolated by using magnetic beads extracted from 40 HCC patients and healthy controls. YAP-1 expression only significantly increased in T cells, not the monocytes (Fig. $1 \mathrm{~B}, \mathrm{C}$ ). The enrolled HCC patients were divided into two groups (YAP- ${ }^{\text {high }}, \mathrm{n}=101$; and YAP$1^{\text {low }}, n=51$ ) based on the YAP-1 expression in the healthy controls. Among the total of 152 HCC patients, we have 140 patients with follow-up survival data. Survival rate analysis was performed in two groups, the 5-year survival rate in the YAP- $1^{\text {high }}$ group $(n=94)$ was low at $11.77 \%$, and significantly different from the YAP- $1^{\text {low }}$ group $(n=46)$ at $42.3 \%$ (HR: $2.059,95 \%$ CI: 1.158-2.054, P=0.0004) (Fig. 1D).

YAP-1 is statistically correlated to Treg percentage within the PBMCs of HCC patients

The expression and activation of YAP-1 was detected within HCC tissues by using its activation by phosphorylation at S127, which was overexpressed in the infiltrated immune cells of HCC tissues, and moreover its expression was associated with Foxp3 expression (Fig. $2 \mathrm{~A})$. To verify the hypothesis that overexpression and activation of YAP-1 within T cells might be related to the percentage of Tregs in HCC patients, the percentage of Tregs was determined in the PBMCs. The YAP- $1^{\text {high }}$ patients had a significantly higher Treg percentage than the YAP$1^{\text {low }}$ patients (Fig. 2B). The activation of YAP-1 was also more apparent in YAP- $1^{\text {high }}$ patients with stronger expression of Foxp3 than the YAP- $1^{\text {low }}$ patients (Fig. 2C). Moreover, the YAP-1 expression in the PBMCs was linearly positively correlated to Treg percentage $\left(\mathrm{R}^{2}=0.1556\right.$, $\mathrm{P}=0.0012$ ) (Fig. 2D).

\section{KARGER}


Fig. 1. YAP-1 was overexpressed in the peripheral $\mathrm{T}$ cells and acted as an index for a bad HCC prognosis. A: The expression of YAP1 was determined by real-time PCR within the PBMCs isolated from HCC patients $(n=152)$ and healthy controls $(n=91)$. B and C: The expression of YAP-1 was determined by real-time PCR within $\mathrm{T}$ cells and monocytes, respectively, which were isolated from the HCC patients and healthy controls $(n=40)$. D: The overall survival rate was evaluated using the Kaplan-Meier curve in the YAP-1 $1^{\text {high }}(n=94)$ and YAP- $1^{\text {low }}(n=46)$ groups. The data were presented as mean \pm SD.* $\mathrm{P}<0.05$; ** $\mathrm{P}<0.01$.

Fig. 2. YAP-1 was statistically correlated to Treg percentage within the PBMCs of HCC patients. A: Representative Figures of IHC staining for YAP-1 (S127) and Foxp3 in the infiltrated immune cells within HCC in the YAP- $1^{\text {high }}$ and YAP-1 ${ }^{\text {low }}$ groups. B: The comparison of Tregs (CD4+CD25+Foxp3+ cells) was carried out in the YAP-1 $1^{\text {high }}$ and YAP-1 ${ }^{\text {low }}$ groups by flow cytometry. C: Expression of Foxp3, YAP-1 (S127), and YAP1 in peripheral $\mathrm{T}$ cells within the YAP- $1^{\text {high }}$ and YAP- $1^{\text {low }}$ groups was determined by western blot. D: The correlation between relative expression of YAP-1 expression and Treg percentage within HCC PBMCs was accessed by linear correlation analysis. The data were presented as mean \pm SD.* $\mathrm{P}<0.05$; ${ }^{* *} \mathrm{P}<0.01$.
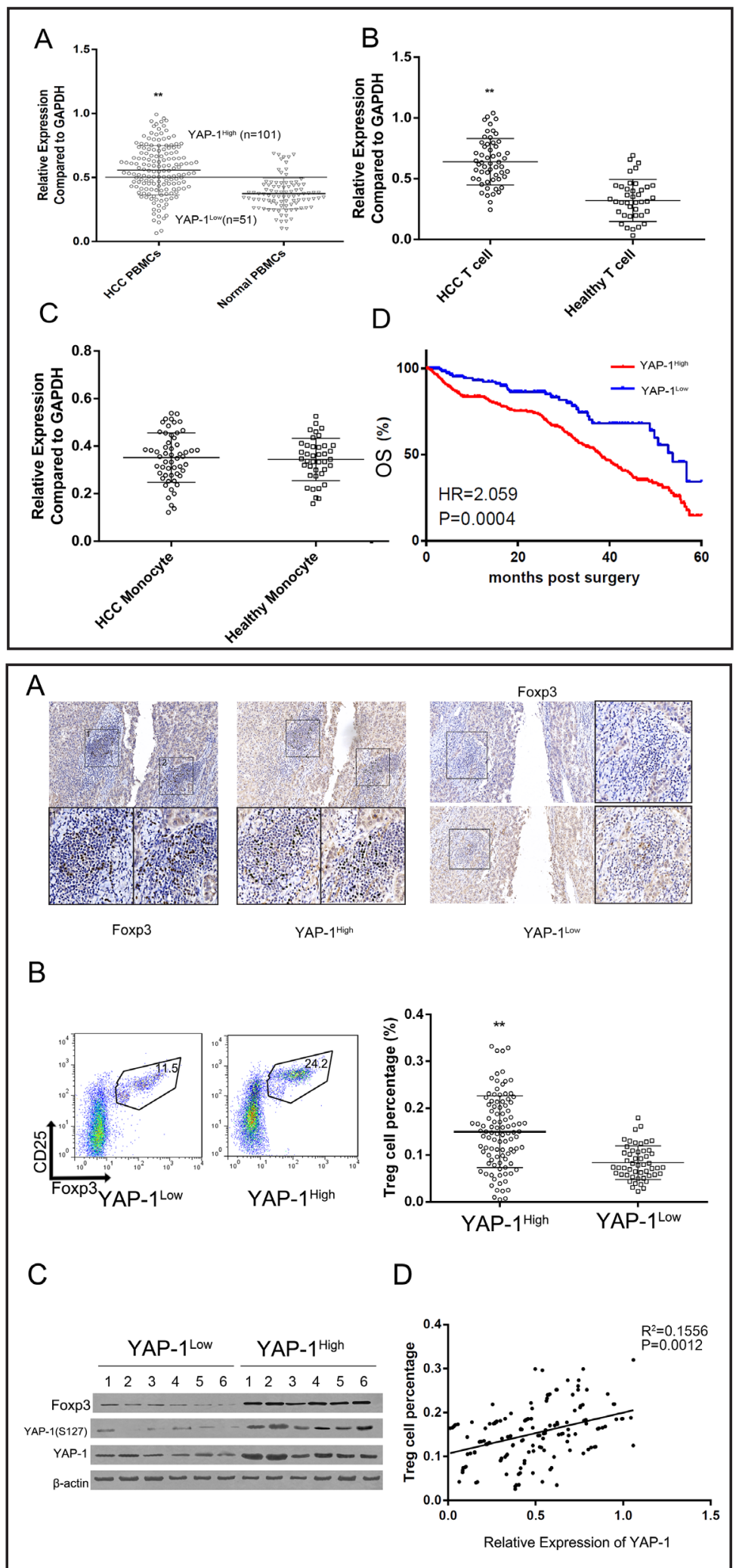

Activation of YAP-1 enhances Treg differentiation by up-regulation of TGFBR2

We also performed in vitro studies to investigate the detailed mechanism of Treg differentiation-promotion affected by YAP-1. The T cells isolated from healthy controls were transduced with YAP-1 and shRNA targeting of YAP-1 (Fig. 3A). Next we determined the 
Fig. 3. Activation of YAP-1 enhances Treg differentiation by up-regulation of TGFBR2. A: YAP-1 expression was determined with different treatment within $\mathrm{T}$ cells. B: The comparison of Treg percentage was carried out in different groups indicated by flow cytometry. C: The mRNA and protein expression of TGFBR2 were accessed in $T$ cells treated differently as indicated in the figure D: The percentage of Tregs was compared in $\mathrm{T}$ cells transduced differently as indicated in the figure. The data were presented as mean \pm SD.* $\mathrm{P}<0.05$; ** $\mathrm{P}<0.01$.

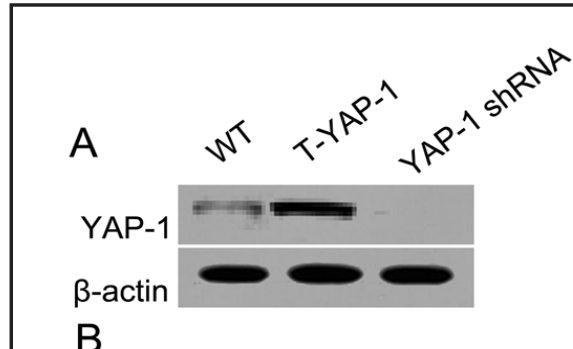

B
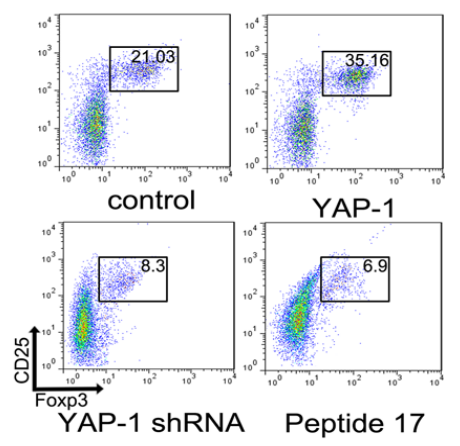

C
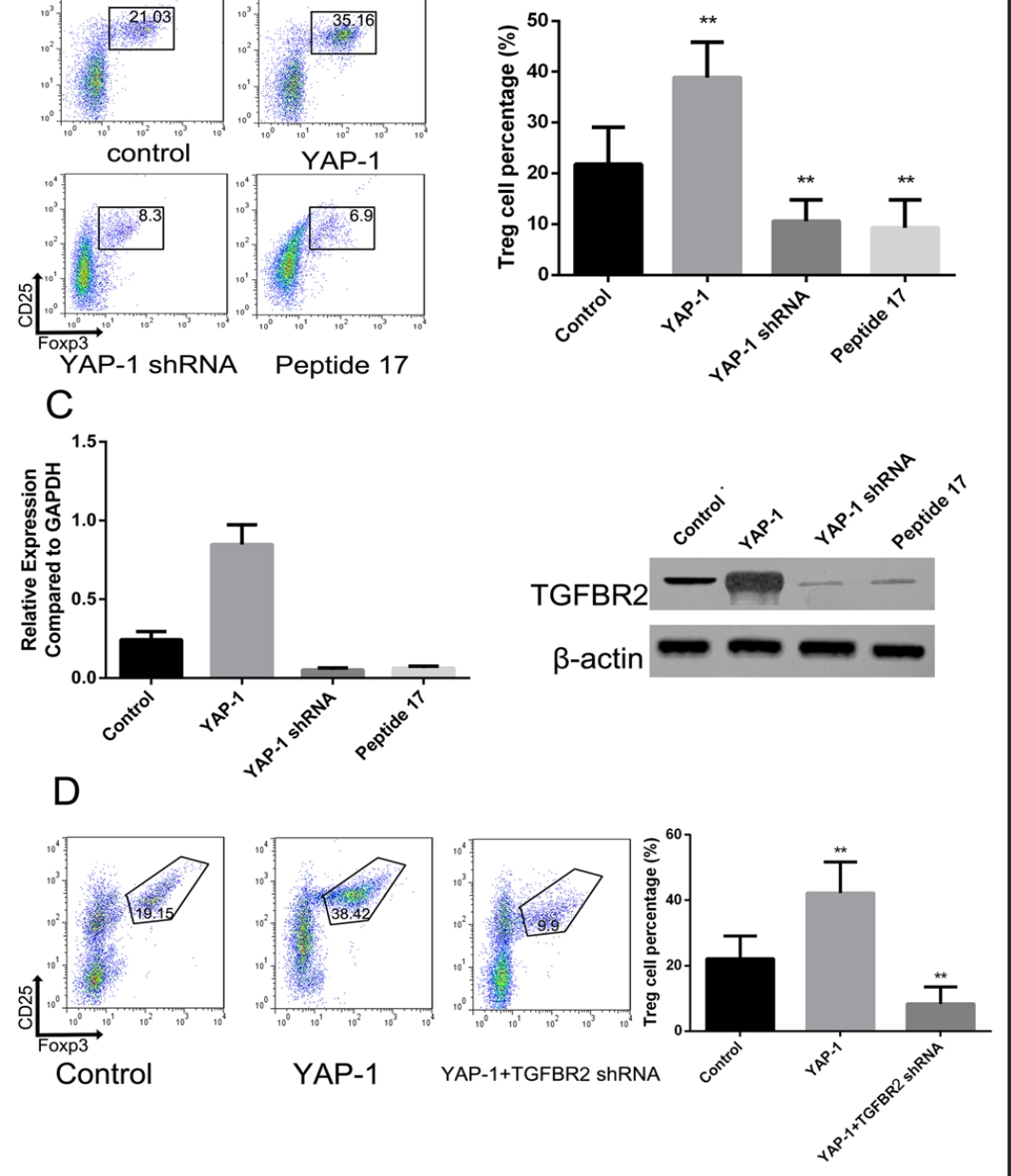

percentage of Tregs with polarization stimulation by using flow cytometry. Overexpression of YAP-1 in T cells can significantly increase the Treg percentage while knockdown of YAP-1 expression decreased the Treg percentage significantly. Also reduce the activation of YAP-1 by using its inhibitor peptide 17 [21] can significantly reduce the percentage of Tregs (Fig. 3B).

TGFBR2 is the receptor for TGF- $\beta$ signal transduction, which is essential for Treg differentiation, and previous studies have revealed that YAP- 1 is related to TGF- $\beta$ signaling [10]. We detected the expression of TGFBR2 within T cells transduced differently. We found that both the mRNA and protein of TGFBR2 increased significantly in T cells with YAP-1 overexpression, while decreased in YAP-1 silenced by its shRNA or its activation was inhibited by peptide 17 (Fig. 3C). Moreover, the specificity of YAP-1 was verified by knockdown TGFBR2 expression in T cells overexpression of YAP-1. Tregs significantly decreased indicating that 
overexpression YAP-1 can promote Treg differentiation specifically by up-regulation of TGFBR2 (Fig. 3D).

Clinical data also support this regulation. We found overexpression of TGFBR2 in HCC PBMCs compared to healthy controls (Fig. 4A). The transcription and protein expression of TGFBR2 was also significantly enhanced within the YAP- $1^{\text {high }}$ group compared to the YAP-1 $1^{\text {low }}$ group (Fig. 4B, C). Moreover, positive linear correlation was also found between TGFBR2 and YAP-1 mRNA expression within the PBMCs of HCC patients $\left(\mathrm{R}^{2}=0.0693\right.$, $\mathrm{P}=0.0006$ ) (Fig. 4D). However, the detailed mechanism remains to be further elucidated.

Up-regulation of TGFBR2 in T cells depends on YAP-1/TEADs at the transcriptional level

To investigate the detailed mechanism of YAP-1 regulation on TGFBR2, DNA binding sites within a 1000 base-pair region upstream of the starting codon "ATG" of TGFBR2 promoter were investigated. Since YAP is a co-activator of TEADs and has no DNA binding site, its regulation on TGFBR2 is TEADs dependent [17]. A TEADs binding site "GGAATG" was detected in the promoter region of TGFBR2 (Fig. 5A). Transcriptional activity significantly decreased when the binding site was deleted. Overexpression of YAP-1 can significantly increase the transcription, but has no apparent effect on transcription activity without the TEADs binding site (Fig. 5B). We used co-IP assay to confirm the interaction of YAP-1 with TEADs in a T cell line, Jurkat (Fig. 5C). The binding of TEADs on the promoter region of TGFBR2 was confirmed by using ChIP-real-time PCR (Fig. 5D), and the results indicated that the YAP-1/TEADs complex could bind to the TGFBR2 promoter in a TEADs DNA binding way.

\section{Discussion}

Physiologically, Tregs comprise diverse subsets of immunosuppressive cells that play critical roles in maintaining immune homeostasis and self-tolerance [22]. However, in cancers, Tregs are able to suppress anti-tumor immune responses and contribute to the development of an immunosuppressive TME, thus promoting
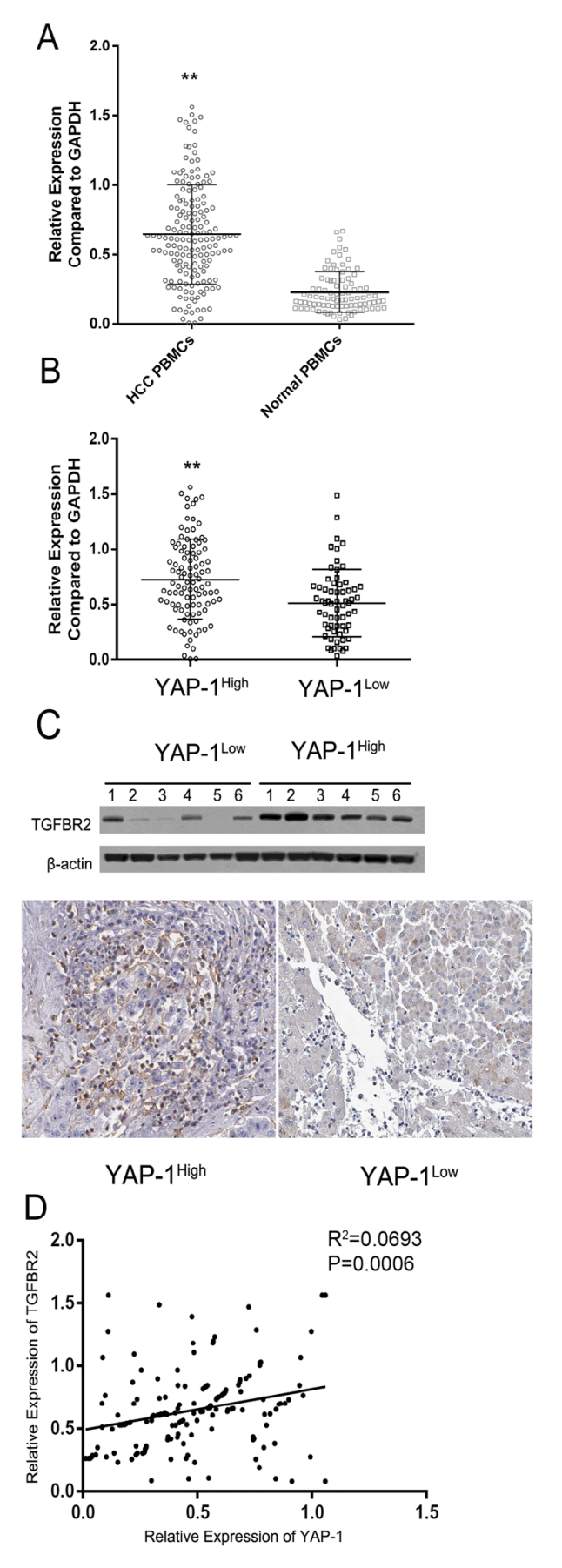

Fig. 4. A: Comparisons of the transcription of TGFBR2 in PBMCs between both HCC patients and healthy controls and YAP- $1^{\text {high }}$ and YAP- $1^{\text {low }}$ were carried out using real-time PCR. B and C: Protein expression of TGFBR2 was determined by western blot and IHC in T cells of the YAP- $1^{\text {high }}$ and YAP- $1^{\text {low }}$ groups. D: The correlation between relative expression of YAP1 and TGFBR2 in the peripheral T cells within HCC was accessed by linear correlation analysis. The data were presented as mean \pm SD. ${ }^{*} \mathrm{P}<0.05$; ${ }^{* *} \mathrm{P}<0.01$. 


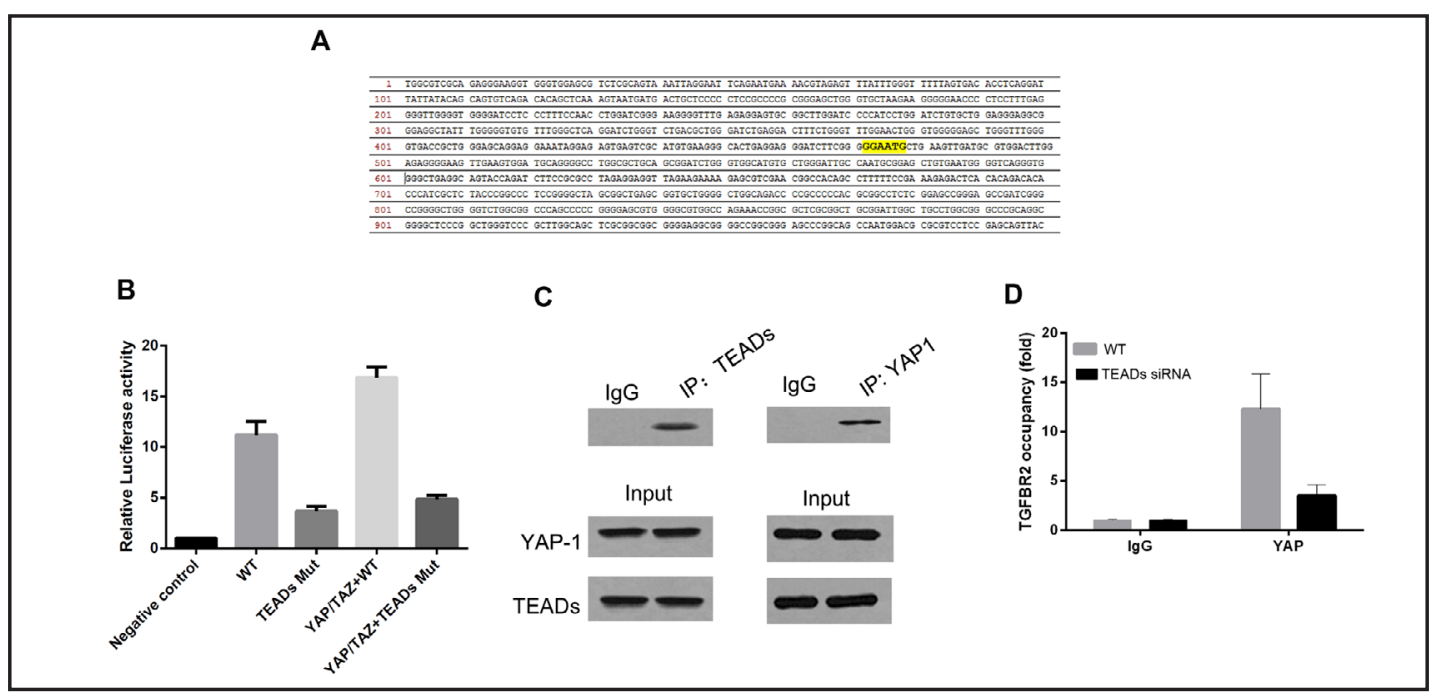

Fig. 5. Up-regulation of TGFBR2 in T cell dependent on YAP-1/TEADs at the transcriptional level. A: Prediction of TEADs-binding DNA sequence was presented within TGFBR2 promoter. B: The promoter activity of different mutants and treatment were accessed by luciferase reporter assay. C: The interaction between YAP-1 and TEADs was confirmed by using co-IP assay. D: The binding of YAP-1/TEADs complex on the TGFBR2 promoter was confirmed by ChIP-real-time PCR. The data were presented as mean $\pm \mathrm{SD}$. ${ }^{*}<0.05$; ** $\mathrm{P}<0.01$.

immune evasion and cancer progression [10]. Previous reports have indicated that TGF- $\beta 1$ and its downstream signaling play an essential role in Treg development [23, 24]. More than 35 TGF family members have been identified, but only five type I and seven type II receptors have been reported [25]. Moreover, studies have further indicated that TGF- $\beta 1$ signaling is transduced mainly through TGFBR2 within T cells $[26,27]$. In the present study, we found that TGFBR2 expression significantly increased within HCC T cells, also related to increased Treg percentage and immunosuppression. TGF- $\beta$ /TGFBR2 signaling might dominantly regulate Treg differentiation. Conditional deletion in T cells of the Tgfbr2 gene can induce inflammatory disorder in mice due to the impaired development of Tregs [28, 29]. Moreover, T-cell-specific ablation of Tgfbr2 also causes T-cell activation and autoimmunity. These TGFBR2-deficient T cells are not suppressed by wild-type Tregs, which suggests that TGF- $\beta$ signaling in effector $\mathrm{T}$ cells is essential for regulation $[28,29]$. Our results indicate, for the first time, that the molecular mechanism of overexpressed TGFBR2 with TME relates to overexpression and activation of YAP-1.

YAP-1 is a transcriptional regulator which can act both as a co-activator and a corepressor, and is the critical downstream regulatory target in the Hippo signaling pathway. And it plays a pivotal role in organ size control and tumor suppression by restricting proliferation and promoting apoptosis [15, 17]. Similarly to TGFBR2, most research concerning YAP-1 has been carried out within tumor cells; the roles of YAP-1 in immune cells were relatively poorly known. There are some reports in the literature about the potential roles of YAP-1 in immunosuppression. Hugo et al. reported that YAP-1 signature enrichment was one of the drivers for immunosuppression through a non-genomic and immune evolution of melanoma [30]. And YAP- 1 also related to increased TGF- $\beta$ within HCC, hyperplasia of oval cells and immature cholangiocytes accompanied by inflammatory cell infiltration and fibrosis occurred within the Mob1a/1b double knockout mice, and this is due to over-activated YAP-1 induced elevation of TGF- $\beta$ [31]. Our study indicated a role of YAP-1 within T cells increasing TGFBR2 expression.

We found overexpression and activation of YAP-1 in T cells of both PBMCs and TILs, and its expression was apparently related to Treg percentage. We found that YAP-1 can 
enhance the transcription of TGFBR2 by direct binding to its promoter via the YAP-1/TEADs complex, and increased expression of YAP-1 within PBMCs could serve as an indicator for the prognosis of HCC patients.

\section{Funding}

This work was supported by grants from the National Natural Science Foundation (Grant Number: 81571559 to C.Z.).

\section{Disclosure Statement}

The authors declared no conflict of interests.

\section{Reference}

1 Drake CG, Lipson EJ, Brahmer JR: Breathing new life into immunotherapy: review of melanoma, lung and kidney cancer. Nat Rev Clin Oncol 2014;11:24-37.

2 Rosenberg SA: Decade in review-cancer immunotherapy: entering the mainstream of cancer treatment. Nat Rev Clin Oncol 2014;11:630-632.

3 Sharma P, Allison JP: Immune checkpoint targeting in cancer therapy: toward combination strategies with curative potential. Cell 2015;161:205-214.

4 Topalian SL, Drake CG, Pardoll DM: Immune checkpoint blockade: a common denominator approach to cancer therapy. Cancer Cell 2015;27:450-461.

5 Chakravarti D, Wong WW: Synthetic biology in cell-based cancer immunotherapy. Trends Biotechnol 2015;33:449-461.

6 Kenderian SS, Ruella M, Gill S, Kalos M: Chimeric antigen receptor T-cell therapy to target hematologic malignancies. Cancer Res 2014;74:6383-6389.

7 Restifo NP, Dudley ME, Rosenberg SA: Adoptive immunotherapy for cancer: harnessing the T cell response. Nat Rev Immunol 2012;12:269-281.

8 Tel J, van der Leun AM, Figdor CG, Torensma R, de Vries IJ: Harnessing human plasmacytoid dendritic cells as professional APCs. Cancer Immunol Immunother 2012;61:1279-1288.

-9 Joshi NS, Akama-Garren EH, Lu Y, Lee DY, Chang GP, Li A, DuPage M, Tammela T, Kerper NR, Farago AF, Robbins R, Crowley DM, Bronson RT, Jacks T: Regulatory T Cells in Tumor-Associated Tertiary Lymphoid Structures Suppress Anti-tumor T Cell Responses. Immunity 2015;43:579-590.

10 Ha TY: The role of regulatory T cells in cancer. Immune Netw 2009;9:209-235.

11 Yang XH, Yamagiwa S, Ichida T, Matsuda Y, Sugahara S, Watanabe H, Sato Y, Abo T, Horwitz DA, Aoyagi Y: Increase of CD4+ CD25+ regulatory T-cells in the liver of patients with hepatocellular carcinoma. J Hepatol 2006;45:254-262.

12 Fu J, Xu D, Liu Z, Shi M, Zhao P, Fu B, Zhang Z, Yang H, Zhang H, Zhou C, Yao J, Jin L, Wang H, Yang Y, Fu YX, Wang FS: Increased regulatory T cells correlate with CD8 T-cell impairment and poor survival in hepatocellular carcinoma patients. Gastroenterology 2007;132:2328-2339.

$>13$ Gao Q Qiu SJ, Fan J, Zhou J, Wang XY, Xiao YS, Xu Y, Li YW, Tang ZY: Intratumoral balance of regulatory and cytotoxic T cells is associated with prognosis of hepatocellular carcinoma after resection. J Clin Oncol 2007;25:2586-2593.

14 Wang Y, Liu T, Tang W, Deng B, Chen Y, Zhu J, Shen X: Hepatocellular Carcinoma Cells Induce Regulatory T Cells and Lead to Poor Prognosis via Production of Transforming Growth Factor-beta1. Cell Physiol Biochem 2016;38:306-318.

15 Zanconato F, Cordenonsi M, Piccolo S: YAP/TAZ at the Roots of Cancer. Cancer Cell 2016;29:783-803.

16 Yap TA, Smith AD, Ferraldeschi R, Al-Lazikani B, Workman P, de Bono JS: Drug discovery in advanced prostate cancer: translating biology into therapy. Nat Rev Drug Discov 2016;15:699-718. 


\section{Cellular Physiology Cell Physiol Biochem 2017;41:1189-1198

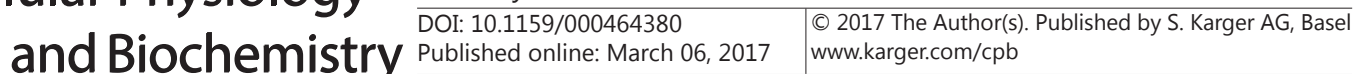

Fan et al.: YAP-1 Promote Differentiation of Tregs

17 Zanconato F, Battilana G, Cordenonsi M, Piccolo S: YAP/TAZ as therapeutic targets in cancer. Curr Opin Pharmacol 2016;29:26-33.

18 Kanai F, Marignani PA, Sarbassova D, Yagi R, Hall RA, Donowitz M, Hisaminato A, Fujiwara T, Ito Y, Cantley LC, Yaffe MB: TAZ: a novel transcriptional co-activator regulated by interactions with 14-3-3 and PDZ domain proteins. EMBO J 2000;19:6778-6791.

19 Qin F, Tian J, Zhou D, Chen L: Mst1 and Mst2 kinases: regulations and diseases. Cell Biosci 2013;3:31.

-20 Wang G, Lu X, Dey P, Deng P, Wu CC, Jiang S, Fang Z, Zhao K, Konaparthi R, Hua S, Zhang J, Li-Ning-Tapia EM, Kapoor A, Wu CJ, Patel NB, Guo Z, Ramamoorthy V, Tieu TN, Heffernan T, Zhao D, Shang X, Khadka S, Hou P, Hu B, Jin EJ, Yao W, Pan X, Ding Z, Shi Y, Li L, Chang Q, Troncoso P, Logothetis CJ, McArthur MJ, Chin L, Wang YA, DePinho RA: Targeting YAP-Dependent MDSC Infiltration Impairs Tumor Progression. Cancer Discov 2016;6:80-95.

-21 Zhang Z, Lin Z, Zhou Z, Shen HC, Yan SF, Mayweg AV, Xu Z, Qin N, Wong JC, Zhang Z, Rong Y, Fry DC, Hu T: Structure-Based Design and Synthesis of Potent Cyclic Peptides Inhibiting the YAP-TEAD Protein-Protein Interaction. ACS Med Chem Lett 2014;5:993-998.

22 Vignali DA, Collison LW, Workman CJ: How regulatory T cells work. Nat Rev Immunol 2008;8:523-532.

-23 Ruegemer JJ, Ho SN, Augustine JA, Schlager JW, Bell MP, McKean DJ, Abraham RT: Regulatory effects of transforming growth factor-beta on IL-2- and IL-4-dependent T cell-cycle progression. J Immunol 1990;144:1767-1776.

24 Shen Y, Wei Y, Wang Z, Jing Y, He H, Yuan J, Li R, Zhao Q Wei L, Yang T, Lu J: TGF-beta regulates hepatocellular carcinoma progression by inducing Treg cell polarization. Cell Physiol Biochem 2015;35:1623-1632.

-25 Hata A, Chen YG: TGF-beta Signaling from Receptors to Smads. Cold Spring Harb Perspect Biol DOI:10.1101/cshperspect.a022061.

-26 Liu Y, Xu Y, Sun J, Ma A, Zhang F, Xia S, Xu G, Liu Y: AKT hyperactivation confers a Th1 phenotype in thymic Treg cells deficient in TGF-beta receptor II signaling. Eur J Immunol 2014;44:521-532.

-27 Ramalingam R, Larmonier CB, Thurston RD, Midura-Kiela MT, Zheng SG, Ghishan FK, Kiela PR: Dendritic cell-specific disruption of TGF-beta receptor II leads to altered regulatory T cell phenotype and spontaneous multiorgan autoimmunity. J Immunol 2012;189:3878-3893.

-28 Li MO, Sanjabi S, Flavell RA: Transforming growth factor-beta controls development, homeostasis, and tolerance of $\mathrm{T}$ cells by regulatory $\mathrm{T}$ cell-dependent and -independent mechanisms. Immunity 2006;25:455471.

29 Marie JC, Liggitt D, Rudensky AY: Cellular mechanisms of fatal early-onset autoimmunity in mice with the T cell-specific targeting of transforming growth factor-beta receptor. Immunity 2006;25:441-454.

-30 Hugo W, Shi H, Sun L, Piva M, Song C, Kong X, Moriceau G, Hong A, Dahlman KB, Johnson DB, Sosman JA, Ribas A, Lo RS: Non-genomic and Immune Evolution of Melanoma Acquiring MAPKi Resistance. Cell 2015;162:1271-1285.

-31 Nishio M, Sugimachi K, Goto H, Wang J, Morikawa T, Miyachi Y, Takano Y, Hikasa H, Itoh T, Suzuki SO, Kurihara H, Aishima S, Leask A, Sasaki T, Nakano T, Nishina H, Nishikawa Y, Sekido Y, Nakao K, Shin-Ya K, Mimori K, Suzuki A: Dysregulated YAP1/TAZ and TGF-beta signaling mediate hepatocarcinogenesis in Mob1a/1b-deficient mice. Proc Natl Acad Sci USA 2016;113:E71-80. 